\title{
Psychophysiological Monitorization in a Special Operation Selection Course
}

Alberto J. Hormeño-HolgadoVicente J. Clemente-Suárez

\section{Abstract}

The present research aimed: i. to analyse the psychophysiological response of soldiers undertaking a special operation selection course; ii. to study the relationship between fat and muscle loss and the psychophysiological response of soldiers undertaking a special operation selection course. We analysed 46 professional soldiers from a special operations unit $(25.1 \pm 5.0$ years, $1.8 \pm 0.1 \mathrm{~cm}$, $76.8 \pm 7.9 \mathrm{~kg}, 24.4 \pm 2.5 \mathrm{~kg} / \mathrm{m} 2$ ) undertaking the last phase of their 10 weeks special operation selection course. Before and immediately after the exercise the following variables were assessed: Stress subjective perception, fatigue subjective perception, rating of perceived perception, cortical arousal, body temperature, blood oxygen saturation, spirometry, isometric hand strength, lower body muscular strength, urine, body composition, life engagement test, coping flexibility scale, acceptance and action questionnaire, perceived stress scale, anxiety state, visual analogue scale and differential aptitude test. A special operation selection course induced an intense stress and physical response as suggested by the psychophysiological changes with a significant $(p<0.05)$ increase in fatigue and stress subjective perception, blood oxygen saturation, $\mathrm{Ph}$, cognitive impairment and motivation-loss. Moreover, decreased leg strength, peak expiratory flow, cortical arousal, body composition, body weight, fat and muscle mass, anxiety stress, alertness, sadness and tension decreased after the exercise. Regarding body composition, higher muscle mass loss participants were related to a higher cognitive impairment and similar psychophysiological response than lower fat mass loss participants.

Keywords:

Cortical arousal, Stress, Anxiety, Military, Sleep deprivation 